\title{
Reconsolidation from negative emotional pictures: Is successful retrieval required?
}

\author{
Bridgid Finn • Henry L. Roediger III • \\ Emily Rosenzweig
}

Published online: 4 April 2012

(C) Psychonomic Society, Inc. 2012

\begin{abstract}
Finn and Roediger (Psychological Science 22:781$786,2011)$ found that when a negative emotional picture was presented immediately after a successful retrieval, later test performance was enhanced as compared to when a neutral picture or a blank screen had been shown. This finding implicates the period immediately following retrieval as playing an important role in determining later retention via reconsolidation. In two new experiments, we investigated whether successful retrieval was required to show the enhancing effect of negative emotion on later recall. In both experiments, the participants studied Swahili-English vocabulary pairs, took an intervening cued-recall test, and were given a final cuedrecall test on all items. In Experiment 1, we tested a distinctiveness explanation of the effect. The results showed that neither presentation of a negative picture just prior to successful retrieval nor presentation of a positive picture after successful retrieval produced the enhancing effect that was seen when negative pictures were presented after successful retrieval. In Experiment 2, we tested whether the enhancing effect would occur when a negative picture followed an unsuccessful retrieval attempt with feedback, and a larger enhancement effect occurred after errors of commission than after errors of omission. These results indicate that effort in retrieving is critical to the enhancing effect shown with negative pictures; whether the target is produced by the participant or given by an external source following a commission error does not matter. We interpret these results as support for semantic enrichment as a key
\end{abstract}

B. Finn $(\bowtie) \cdot$ H. L. Roediger III $\cdot$ E. Rosenzweig Department of Psychology, Washington University in St. Louis, Campus Box 1125, One Brookings Drive,

St. Louis, MO 63130, USA

e-mail: bridgid.finn@wustl.edu element in producing the enhancing effect of negative pictures that are presented after a retrieval attempt.

Keywords Memory retrieval $\cdot$ Reconsolidation $\cdot$ Emotion and memory

Understanding the dynamic nature of retrieval is a core issue for memory research. We focus here on the finding that retrieval can modify memory and promote long-term learning. Research has shown that retrieval practice has positive effects on learning that outweigh those gained from a representation of the same material. The fact that testing can enhance learning has been called the testing effect (e.g., Carrier \& Pashler, 1992; Roediger \& Karpicke, 2006). The view of tests as memory modifiers (Bjork, 1975) has important implications for education. Rather than being seen purely as an instrument to assess what a student knows, tests are being more widely acknowledged as learning events and touted as effective tools for studying (e.g., McDaniel, Roediger, \& McDermott, 2007). In the present experiments, we explored how processing that occurs in the period immediately after retrieval affects the benefits that result from testing (Finn \& Roediger, 2011).

Several theoretical accounts of testing effects (see Roediger \& Butler, 2011, for a recent review) have identified the processing involved in the retrieval attempt itself as the source of the enhancement (Bjork, 1975; Jacoby, Shimizu, Daniels, \& Rhodes, 2005; McDaniel \& Masson, 1985). Bjork, for example, argued that retrieval, particularly when it is effortful, involves elaborative, deep processing of the information, which will thereby enhance later retention (Craik \& Tulving, 1975). A study by Gardiner, Craik, and Bleasdale (1973) supported this claim. They measured the time that it took participants to retrieve responses on a free 
recall test and used these latencies as indices of retrieval effort. Their results showed that items that took longer to retrieve on a first test were more likely to be recalled on a follow-up test than were items that were retrieved more quickly (see also Benjamin, Bjork, \& Schwartz, 1998; Carpenter \& DeLosh, 2005; Glover, 1989).

\section{A role for postretrieval processing in the testing effect}

Recently, Finn and Roediger (2011) demonstrated that processes during the period immediately subsequent to successful retrieval can also play an important role in later retention. In their study, participants studied Swahili-English translations (e.g., mashua-boat) and then took a cued-recall test. Upon the successful retrieval of the English translation during the test, a blank screen, a neutral picture, or a negatively valenced arousing picture was presented. The results showed that when a negative picture followed successful retrieval (either immediately or after a 2-s delay), recall on a final test was enhanced as compared to when retrieval had been followed by a blank screen or a neutral picture. Critically, when a negative picture was presented following a restudy opportunity, the enhancing effect was absent. Thus, the results implicated the importance of postretrieval processing in augmenting the standard testing effect, because a manipulation introduced after successful retrieval of a target amplified the effect.

The Finn and Roediger (2011) findings are broadly consistent with a body of work supporting the concept of reconsolidation. Reconsolidation refers to the idea that when information is retrieved from memory, it enters a labile state rendering it amenable to change (for a review, see, e.g., Dudai, 2004). Reconsolidation triggered by reactivation of a memory is thought to be an important component of longterm memory processing (Dudai, 2006; Lewis, 1979; Nader, 2003; Nader, Schafe, \& LeDoux, 2000; Tronson \& Taylor, 2007), with its functional role being to strengthen the reactivated trace and/or to update it with new information (see Sara, 2000a, for a review). While the conceptualization of reconsolidation is still under debate, most theories agree that a memory can be modified in the period following its retrieval (see, e.g., Tronson \& Taylor, 2007, for a review). Of course, a long tradition in experimental psychology has also shown both negative effects (retroactive interference and misinformation effects) and positive effects (testing effects) from the act of retrieval (e.g., Barnes \& Underwood, 1959; Carrier \& Pashler, 1992; Loftus \& Palmer, 1974).

In Finn and Roediger's (2011) experiments demonstrating the enhancing effect, a negative picture was presented immediately following retrieval - that is, during the period of time in which the reconsolidation hypothesis posits that a memory trace is labile. The negative-picture manipulation may have enhanced later retention relative to the neutral and blank conditions because the pictures aroused negative emotion, and the emotional centers of the brain (primarily the amygdala) are closely interconnected with the hippocampus, an area involved in consolidation and reconsolidation (see Cahill \& McGaugh, 1998; Kensinger, 2009). Amygdalamediated effects of negative emotional arousal on memory are thought to involve the activation of stress hormone systems that are implicated in memory regulation (McGaugh, 2000; McGaugh \& Roozendaal, 2002; Roozendaal, Barsegyan, \& Lee, 2008).

The bulk of the experiments that have targeted the reconsolidation process have used postretrieval treatments in animal models. Most studies have demonstrated impairments in retention when amnestic agents are administered following retrieval or reactivation of a trace (for a review, see, e.g., Dudai, 2006). Several studies have also shown enhancement when agents such as strychnine or amphetamine are administered after retrieval (Blaiss \& Janak, 2006; DeVietti, Conger, \& Kirkpatrick, 1977; Gordon \& Spear, 1973; Inda, Muravieva, \& Alberini, 2011; Lee, Milton, \& Everitt, 2006; Rohrbaugh \& Riccio, 1970; Shema et al., 2011; Tronson \& Taylor, 2007; and see also Frenkel, Maldonado, \& Delorenz, 2005; Frenkel, Suarez, Maldonado, \& Delorenzi, 2010; Rodriguez, Horne, \& Padilla, 1999, for more naturalistic interventions).

Studies targeting retrieval-induced reconsolidation in humans (e.g., Forcato et al., 2007; Hupbach, Gomez, Hardt, \& Nadel, 2007; Schiller et al., 2010) have generally used paradigms that involved impairing retention of the original episode via some experimental manipulation. Finn and Roediger's (2011) findings and those from a study by Coccoz, Maldonado, and Delorenzi (2011) have recently shown that a manipulation following retrieval can enhance long-term retention of human episodic memory. Coccoz et al. demonstrated that when participants were exposed to a mildly painful coldwater stressor immediately after reactivation of a memory, later retention benefited. Exposure to warm water following reactivation of the trace, as well as exposure to the cold-water stressor without reactivation, did not produce enhancement. These findings converge with those of Finn and Roediger and show that mild stress and/or negative arousal following retrieval can enhance long-term retention.

\section{The present studies}

In the Finn and Roediger (2011) experiments, active retrieval seemed necessary to demonstrate the enhancing effect of negative pictures on later recall, since the manipulation was effective when it followed successful retrieval but was ineffective when it followed a restudy presentation. The primary question addressed in the present experiments is whether a 
retrieval attempt must end successfully for the enhancing effect of negative pictures to emerge. In two experiments, we varied when an emotionally valenced picture was presented during the retrieval process to test the hypothesis that an active and effortful (but not necessarily successful) retrieval attempt must occur for arousal to enhance later recall. To this end, we compared manipulations of presenting negative pictures, positive pictures, neutral pictures, or blank slides before successful retrieval, after successful retrieval, and after an unsuccessful retrieval attempt with feedback. If positive pictures produce the same effect as negative pictures, we will have shown that negative arousal is not the only emotional effect producing the enhancing effect. If negative and positive pictures produce an enhancing effect when presented before retrieval of the critical target, it would be possible that the enhancing effect results from distinctiveness or isolation rather than via emotion-induced reconsolidation.

In Experiment 1, we compared a preretrieval condition to two postretrieval conditions in which negatively and positively valenced pictures were presented after retrieval. Of course, we expected to replicate the enhancing effect of the negative pictures after successful retrieval on later retention (as in Finn \& Roediger, 2011). We predicted that presenting negative pictures before successful retrieval would have no effect, because the memory had not yet been reactivated. Introduction of the enhancing or disrupting manipulation immediately after retrieval has been critical to tests of the reconsolidation hypothesis, which posits that retrieval induces a time-limited period of lability during which memory modification can occur (e.g., Tronson \& Taylor, 2007). In many of the studies reported in the literature, presentation of the manipulating event outside of the short period of time following reactivation has served as the control condition that demonstrated that a reconsolidation manipulation must occur soon after the retrieval or reactivation of a trace for memory modification to occur (e.g., Coccoz et al., 2011; Nader et al., 2000; Przybyslawski \& Sara, 1997).

If a retrieval attempt is critical to show the enhancing effect of emotion on later recall, an emotional picture presented before retrieval has begun should have no impact on later retention. Such a finding would provide additional support that the period following a retrieval attempt (thought to involve reconsolidation) is the route by which negative emotion conveys its advantage on later retention. However, if the negative picture has a positive effect not because of reconsolidation but because its close temporal relation to the target event makes that event more distinctive, presenting the picture before retrieval of the target event should also boost later retention.

Experiment 1 also included a condition in which positive pictures were presented after successful retrieval, which (a) allowed for an examination of whether another distinct valence condition would give rise to the enhancing effect and (b) provided an additional test of the distinctiveness account. Further evidence against a distinctiveness interpretation would be provided if the enhancing effect did not occur with positive pictures that were matched to the negative pictures for arousal and presentation frequency. Accordingly, Experiment 1 was designed to test between the reconsolidation and distinctiveness accounts.

Experiment 2 was aimed at the more interesting case of the effects of a negative picture following unsuccessful retrieval of the target, coupled with feedback. Would the enhancement occur if participants attempted to retrieve targets, failed, and were then given feedback? In addition, errors may occur in two ways, by commission (giving a wrong response) or omission (giving no response). The former presumably permits retrieval effort and semantic processing, even if it results in the wrong target being retrieved, and the latter may or may not involve effortful retrieval (e.g., if the subject fails to even recognize the cue, no retrieval attempt may be made). If retrieval effort is partly responsible for the effect, we might expect a greater effect in cases of errors of commission relative to errors of omission. On the other hand, the very production of an error may create an associative bond to the cue and lead to interference rather than enhancement.

\section{Experiment 1}

To test the reconsolidation hypothesis against a distinctiveness account, we contrasted three conditions in Experiment 1: We presented a negative picture prior to a successful retrieval or after successful retrieval, and the third condition involved presenting a positive picture after successful retrieval. We refer to the condition in which the negative picture occurred before successful retrieval as the preretrieval condition and the conditions in which negative and positive pictures occurred after a successful retrieval as the negative postretrieval and positive postretrieval conditions, respectively.

Experiment 1 allowed for two opportunities of testing a distinctiveness-based explanation of the effect (see Finn \& Roediger, 2011, for a discussion). According to this hypothesis, because it is presented only once in the list, the emotional picture may serve to isolate the critical pair presented before the picture occurs. Such effects often occur in listlearning experiments (Hunt, 2006; Schmidt, 1991, 2002), so ruling out this interpretation is critical to understanding the effect. Finn and Roediger (2011) argued that the effects that they observed did not arise because of the distinctiveness of the negative pictures, because when the negative picture was presented after a pair was restudied (rather than retrieved), no facilitation occurred. Nonetheless, because the issue is so 
important, we provided two further tests of the isolation hypothesis in this experiment.

The preretrieval condition used in Experiment 1 provided one test of a distinctiveness-based explanation. If the negative picture simply serves to make a pair more distinctive, it should produce the effect when it is presented just before the pair is tested, as well as when it is presented just afterward. On the other hand, if the negative picture only produces a positive effect when it occurs after retrieval, in line with the reconsolidation account, reconsolidation rather than distinctiveness would be implicated as the mechanism involved.

The postretrieval presentation of positive pictures provided a second test of the distinctiveness explanation. If positive pictures, which were matched to the negative pictures for arousal ratings and presentation frequency, did not give rise to the enhancing effect, this outcome would provide further evidence that distinctiveness is not a satisfactory explanation for our findings. Of course, it might turn out that positive pictures also produce enhancement, as negative pictures do, in which case the outcome could be due to either reconsolidation or distinctiveness.

\section{Method}

Participants A group of 180 Washington University undergraduate students participated in Experiment 1 for course credit or payment. Nine of the participants were excluded from all of the analyses that follow for failing to answer any items correctly on either the initial or the final test, and one participant was excluded for texting throughout the experiment, leaving the data for 170 participants. In this experiment and the one that follows, all participants were treated according to the ethical guidelines of the American Psychological Association.

Design For this experiment we used a 3 (retrieval condition: preretrieval, negative postretrieval, or positive postretrieval) $\times 3$ (picture event type: blank screen, neutral picture, or negative picture) mixed-measures design. The first variable was manipulated between subjects (with 57 participants in the preretrieval condition, 53 in the negative postretrieval condition, and 60 in the positive postretrieval condition), and the second variable was manipulated within subjects. In the preretrieval condition, the picture manipulation occurred before presentation of the cue for retrieval, and in the postretrieval conditions, the picture manipulation occurred after a retrieval attempt had been made. The primary dependent variable of interest was recall on a final cued-recall test following these three manipulations.

Materials The study materials were composed of 100 pairs of Swahili-English vocabulary words that ranged from difficult (ubini-forgery) to easy (lulu-pearl), as established by the norms of Nelson and Dunlosky (1994). The pairs were randomly assigned to one of ten study lists. The picture stimuli that were presented before and after the retrieval attempt were taken from the International Affective Picture System (IAPS; Lang, Bradley, \& Cuthbert, 2008); this system uses a 9-point rating scale in which higher values indicate more positive values when the pictures are rated on valence or arousal. The negatively valenced pictures that we used (e.g., dead cat or pointed gun) had a mean valence of 2.70 (range: 4.18-1.79) and a mean arousal rating of 5.98 (range: 6.90-5.00). The positively valenced pictures (e.g., sky diving or water skiing) had a higher mean valence, of 7.53 (range: 8.10-7.09), and a mean arousal rating of 6.64 (range: 7.35-6.00). The maximum range for arousal ratings for the negative pictures reflected the constraints of our institutional review board, which required us to eliminate some of the most disturbing images (e.g., mutilated corpses). The neutral pictures (e.g., shoelaces or a fork) included items from the IAPS system as well as items from a set that had been developed for use in other studies and normed using the Lang et al. procedure; their corresponding arousal and valence ratings were 3.53 and 5.74 (with both ratings ranging from 1.00 to 9.00 )

Procedure The participants were told at the start of the experiment that they would study 10 lists of 10 pairs of Swahili-English vocabulary pairs and, after each list, take a test on all 10, with the left-hand member of the pair presented as a cue for recall of the right-hand member. Furthermore, they were told that all 100 pairs would be tested again at the end of the experiment. Participants were also told that as they took the initial test on the 10-item list, they would be presented with a list of pictures that they should also study for a later test (they were not ever tested on the pictures). They were told that some of the pictures might be emotionally salient, and that if they were feeling uncomfortable they were free to leave at any time. The words pairs were randomly assigned to one of the 10 lists, and each pair was presented for $2 \mathrm{~s}$. After studying the 10 pairs, participants solved multiplication problems for $1 \mathrm{~min}$ to remove any effects of short-term memory (Glanzer \& Cunitz, 1966). They then took an intervening cued-recall test on the 10 studied pairs in random order; for each item the Swahili term was presented, and their task was to type in the English translation. The participants typed in their responses and hit Enter when they were done. They were not forced to supply an answer (i.e., they could leave the response field blank), and they had as much time as they wanted to enter their response.

For participants in in the preretrieval condition, a blank screen, a neutral picture, or a negative emotional picture was presented immediately prior to presentation of the cue that 
prompted target recall for a randomly selected pair in each of the 10 lists. The assignment of conditions to item pairs was random. Because the picture manipulation occurred immediately before an item was tested, we could not guarantee that a negative emotional picture would only be presented prior to successful test trials. Thus, on some of the intervening test trials, a negative picture preceded an unsuccessful retrieval attempt. However, final test performance was only evaluated using items that had been successfully retrieved on the intervening test. For the two groups of subjects in the postretrieval conditions, the picture manipulation occurred immediately after a retrieval had been attempted, and the participant hit the Enter button. After a successful retrieval, a blank screen, a neutral picture, or an emotional picture (negatively or positively valenced, according to condition) was presented. Incorrect items were followed by either a blank screen or a neutral picture. The assignment of conditions to item pairs was random, with the proviso that a negative or positive picture occurred after one of the successful retrieval attempts in their respective conditions. In the analysis of final test performance, we considered only items that were answered correctly during this initial test.

The blank screen, the neutral picture, or the emotional picture (e.g., a gun pointing at the participant or a highspeed ski jump) was presented for $500 \mathrm{~ms}$. The presentation order of the pictures was randomized over the 10 lists. An emotional picture was presented at most once per 10-item list, following the oddball paradigm (see Strange, Hurlemann, \& Dolan, 2003; Tulving, 1969). If the participant did not answer any items correctly during the intervening test of the 10-item list, no emotional picture was shown. After the initial test phase, participants completed another minute of math before the next set of 10 pairs was presented for study. After all 10 lists had been studied and tested, all 100 items were randomized and presented for a final cued-recall test. The lag to test between an item's presentation in the initial studytest phase and the final test phase was approximately $30 \mathrm{~min}$. No distractor task intervened between the initial study-test phase and the final test phase. The final test began immediately after the last 10-item list had been studied and tested.

\section{Results}

Initial test performance There was no difference between any of the conditions on the initial test, $F(2,167)=1.27$, $p>.05$. Performance for the preretrieval condition was $M=.35, S E=.02$; positive postretrieval performance was $M=.39, S E=.02$; and negative postretrieval performance was $M=.37, S E=.02$.
Final test performance The final test performance was analyzed using only the items that had been correctly retrieved on the initial test, which limited the data to approximately $37 \%$ of the items on the final test. A preliminary analysis found no interactions with normative item difficulty, and thus the following analyses were collapsed over this variable. Recall on the final test for items successfully retrieved on the initial test is shown in Fig. 1. Relative to the blank and neutral-picture control conditions, only one of the three experimental conditions introduced in the initial test produced a positive effect on the final test: the case in which negative pictures had been presented after successful retrieval. Neither the preretrieval condition nor the positive postretrieval condition showed the enhancement that occurred with the negative postretrieval manipulation. This outcome was confirmed by a significant interaction between retrieval condition and picture event type, $F(4,316)=2.58$, $M S E=.04, p<.05, \eta_{\mathrm{p}}{ }^{2}=.03$. (If participants did not have a value in one or more of the within-participant conditions, they were excluded from the analyses. Thus, the degrees of freedom might differ from the total number of participants in this experiment and in the one that follows.) Separate univariate analyses conducted for each retrieval condition confirmed that neither the preretrieval $(F<1)$ nor the positive postretrieval $(F<1)$ condition showed a significant effect of picture event type. As expected, the negative postretrieval condition did show the standard enhancing effect, $F(2,102)=6.44, M S E=.03, p<.01, \eta_{\mathrm{p}}{ }^{2}$ $=.11$.

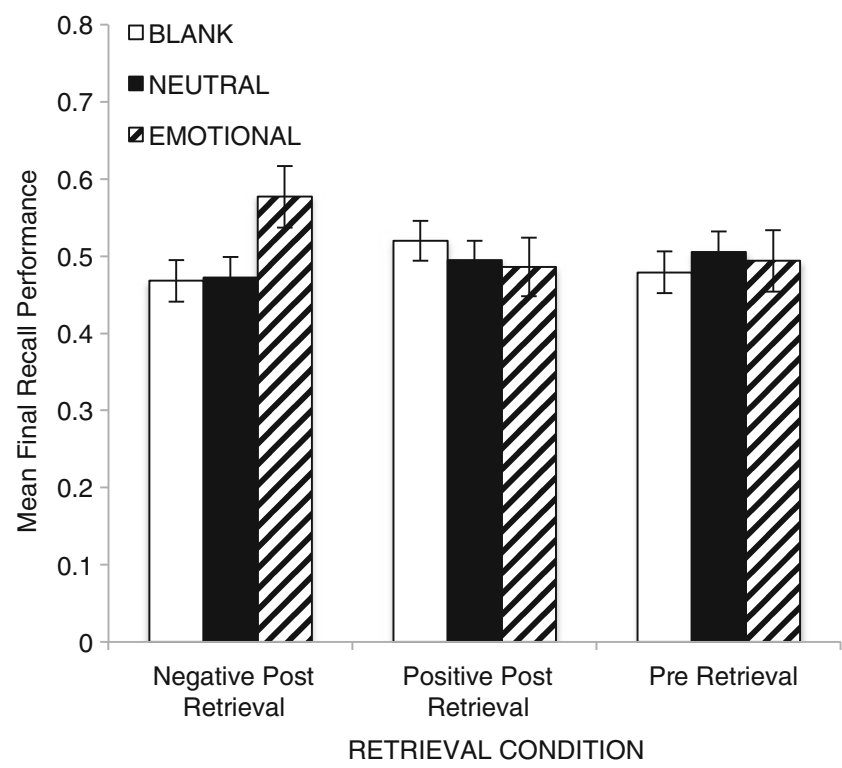

Fig. 1 Mean proportions correct on the final recall test for items answered correctly on the initial test, for each experimental group in Experiment 1. Error bars depict the standard errors of the means 


\section{Discussion}

The data of Experiment 1 provided support for the hypothesis that a retrieval attempt is critical to show the enhancing effect of a negative picture on later recall. Retention was not enhanced when a negative picture was presented just prior to a successful retrieval, but it was enhanced when a negative picture was presented just after a retrieval attempt. This finding is in line with the reconsolidation hypothesis, which posits that memory can be modified during a short period following retrieval.

In addition, the presentation of positively valenced pictures after successful retrieval did not give rise to the effect. In fact, the difference between the positive and blank conditions was in the opposite direction from the one expected, albeit not significantly so. This outcome provides further evidence against distinctiveness underlying the effect, but it leads to a new question: Why did positive pictures fail to produce the same enhancement to later retention as the negative pictures did? While an exhaustive answer to this question is outside the scope of the present article, it is the central topic of a set of studies that are currently in preparation (Finn, Rosenzweig, \& Roediger, 2012). Here, we will speculate only briefly. Even when they are matched for arousal, positive and negative stimuli have been shown to elicit different patterns of behavioral, physiological, and neural responses (Cacioppo \& Gardner, 1999; Carretié, Mercado, Tapia, \& Hinojosa, 2001; Delplanque, Lavoie, Hot, Silvert, \& Sequeira, 2004; Ito, Larsen, Smith, \& Cacioppo, 1998; Kensinger, 2007). For example, the negativity bias refers to the tendency for negative stimuli to elicit more prominent and rapid behavioral responses, which may reflect privileged access to attentional resources (see, e.g., Carretié et al., 2001) or may signal event significance, both of which could have important consequences for the effect we obtained. When retrieval is immediately followed by a significant negative event (i.e., an arousing picture of a gun being pointed at you), enhancing or updating the recently retrieved memory with details from the current retrieval context might be particularly adaptive. However, we hasten to note that neither behavioral nor physiological measures of participant arousal were taken in our study. Thus, we cannot say with confidence that the differences we observed between positive and negative stimuli on our delayed test were due to valence alone, rather than to some other feature. To address this issue, future studies should obtain behavioral and physiological measures of arousal when participants encounter the emotional pictures. The pattern shown with positive pictures does, however, have important implications for the objectives of the present study. The lack of a difference among conditions in the positive condition provides another failure of a distinctiveness account of our findings: A distinct positive picture presented after retrieval did not aid long-term retention.

In sum, the results support the reconsolidation account provided by Finn and Roediger (2011). If the emotional picture serves to isolate certain target pairs and make them memorable because of distinctive processing, providing a negative picture before a pair is successfully retrieved would presumably enhance recall of the pair as well as does providing the picture after retrieval. Likewise, the positive pictures presented after retrieval should have also enhanced later recall. Neither outcome occurred, casting further doubt on a distinctiveness explanation of the findings of enhancement here and in Finn and Roediger's results.

\section{Experiment 2}

Experiment 2 was designed to study whether enhancement from negative pictures would occur in the case of unsuccessful retrieval of a pair when feedback was provided. In the Finn and Roediger (2011) experiments, a blank screen, a negative picture, or a neutral picture was always presented after successful retrieval. Before describing Experiment 2, we will review evidence about the effects of unsuccessful retrieval on performance on later tests.

Disagreement exists regarding the consequences of unsuccessful retrieval attempts on learning - in particular, if commission errors are made. Proponents of errorless learning, Skinner (e.g., 1961) figuring prominently among them, emphasized that learners should avoid guessing because if errors are allowed, the errors will be learned (see Clare \& Jones, 2008, for a review). A related concern is that unsuccessful tests could hamper learning by strengthening incorrect retrieval routes, especially if the participants make errors (e.g., Bjork, 1975; Kornell, Hays, \& Bjork, 2009; McDaniel \& Masson, 1985; but see Carrier \& Pashler, 1992). Studies with patient populations have shown that making errors during learning can have detrimental effects, and errorless learning techniques are effective in promoting memory rehabilitation in individuals with memory impairments (e.g., Baddeley \& Wilson, 1994; Glisky, Schacter, \& Tulving, 1986; see Clare \& Jones, 2008, and Kessels \& de Haan, 2003, for reviews). There is also evidence that making errors can hinder test performance in nonimpaired populations. For example, Hastie, Landsman, and Loftus (1978) showed that when participants were encouraged to guess on a first test, their incorrect answers often appeared on a follow-up test. Similarly, when people make errors during a multiple-choice test, those errors can persist on later cuedrecall tests (Marsh, Roediger, Bjork, \& Bjork, 2007; Roediger \& Marsh, 2005). Using paired-associate learning of digit-CVC pairs (e.g., 5-ZAB), Cunningham and Anderson (1968) showed that when people try, unsuccessfully, 
to retrieve a target (i.e., guess) just prior to the first presentation of that target, learning of the target is diminished relative to when the target is simply presented without a preceding test. Loftus (1996) has written that "guessing can be dangerous" (p. 82) and argued that when people guess, they may later recall their guesses as having been actual events. Indeed, Roediger, Wheeler, and Rajaram (1993) found that when people were forced to guess the answer on a test, they often later remembered their guesses as having been part of the original study list (see also Ackil \& Zaragoza, 1998).

On the other hand, different studies have shown that unsuccessful retrieval can be beneficial to learning, at least as long as the correct answer is ultimately generated by the participant or provided by the experimenter (Begg \& Snider, 1987; Butler, Karpicke, \& Roediger, 2007; Butler \& Roediger, 2007; Carrier \& Pashler, 1992; Finn \& Metcalfe, 2010; Huesler \& Metcalfe, 2010; Izawa, 1971; Kane \& Anderson, 1978; Kornell et al., 2009; Richland, Kornell, \& Kao, 2009; Slamecka \& Fevreiski, 1983). For example, Finn and Metcalfe showed that when unsuccessful retrieval attempts were followed by feedback, long-term retention was better than when the correct answer was simply presented. That is, unsuccessful retrieval attempts that are followed by feedback show a testing effect.

To explain how unsuccessful retrieval attempts could benefit learning, Kornell et al. (2009) proposed that during an unsuccessful attempt, semantically similar concepts are activated, which facilitates subsequent learning of the target item. Supporting this hypothesis, several studies have shown that unsuccessful attempts to retrieve a target facilitate its subsequent learning when the errors are semantically related to the target; however, no such benefit occurs when the errors and the target are unrelated (Begg \& Snider, 1987; Slamecka \& Fevreiski, 1983; see also Grimaldi \& Karpicke, 2010; Heusler \& Metcalfe, 2010). ${ }^{1}$ Activation of related concepts, at least in these paradigms, appears to be a key determinant of whether an unsuccessful retrieval attempt will bolster subsequent learning of the target. Together, these findings suggest that a retrieval attempt, even if unsuccessful, can engage the elaborative processing thought to benefit long-term retention.

Experiment 2 tested the hypothesis that a negative picture presented in the interval following a retrieval attempt will enhance later recall, even when the attempt is unsuccessful. In prior research, the intensity of the reactivation-as measured by the duration of the reactivation session, for example-has been shown to determine whether

\footnotetext{
${ }^{1}$ These studies also help to resolve the apparent conflict between the studies showing benefits from an unsuccessful retrieval and those of Cunningham and Anderson (1968), who used unrelated digit-CVC trigram pairings and did not find that an unsuccessful test benefited learning. The type of materials used probably prevented semantic activation.
}

the reconsolidation process will be initiated (Bustos, Maldonado, \& Molina, 2009; Lee et al., 2006; Suzuki et al., 2004). Accordingly, the amount of effort involved in the retrieval attempt (or the amount of time spent engaging in effortful retrieval) may govern whether enhancement occurs. If a retrieval attempt is effortful enough to trigger reconsolidation, presentation of the negative picture following feedback should give rise to the picture's enhancing effect on later recall. If retrieval is not attempted (i.e., the cue is not recognized and no attempt to retrieve is made), the feedback presentation that follows may function like a restudy opportunity. A large body of research has shown that a restudy presentation does not engage the same elaborative processing as occurs in testing (e.g., Roediger \& Karpicke, 2006). In addition, Finn and Roediger (2011, Exp. 3) previously established that a negative picture does not enhance later recall when it follows a restudy presentation (relative to neutral pictures or blank screens following restudy). Thus, negative emotional pictures may not enhance later recall under all instances of unsuccessful retrieval, such as when the cue is not recognized and/or no serious retrieval attempt occurs.

Experiment 2 investigated whether negative pictures would enhance later recall when they were presented following an unsuccessful retrieval attempt with feedback in the form of the correct answer. After presentation of the feedback, the picture manipulation occurred (depicting a negative emotional picture, a neutral picture, or a blank screen). Final test performance at the end of the session was examined to determine whether negative emotional arousal facilitated later retention, as compared to a neutral picture or a blank screen. Final test performance in the unsuccessful retrieval condition was also compared to performance in a successful retrieval condition, as in Experiment 1. In Experiment 2, for half of the participants, the negative picture, neutral picture, or blank slide occurred after successful retrieval of the target; for the other half of the participants, the negative picture, neutral picture, or blank slide occurred after unsuccessful retrieval followed by feedback. Comparisons between the retrieval conditions permitted assessment of whether attempting retrieval (and receiving feedback) can give rise to the enhancement produced by negative pictures, or whether successful retrieval is necessary to produce the effect. Of course, the effect might occur in both cases, but be magnified by successful retrieval relative to unsuccessful retrieval with feedback. Furthermore, the effect could occur in response to errors of commission and not to errors of omission, because the former case involves elaboration through semantic activation (even if incorrect). Finally, if errors create interference, as has been suggested by some prior work, then the effect may occur after errors of omission (with feedback) and be 
blocked after errors of commission (due to interference). Experiment 2 was aimed at resolving these issues.

\section{Method}

Participants, design, and materials A group of 212 Washington University undergraduate students participated for cash or course credit. One participant who failed to answer any items correctly on the initial test, two participants who failed to complete the experiment, and two participants who were under age 18 were excluded from all analyses, leaving 207 participants with useable data. The experiment involved a 2 (retrieval condition: unsuccessful retrieval with feedback or successful retrieval) $\times 3$ (picture event type: blank screen, neutral picture, or negative picture) mixed-measures design. The first variable was manipulated between subjects (with 104 in the successful retrieval condition and 103 in the unsuccessful retrieval condition), and the second was manipulated within subjects. The study materials were identical to those used in Experiment 1. In addition, in the unsuccessful retrieval condition, the manipulation could follow either a trial with a response omission or a trial with an incorrect response.

Procedure As in Experiment 1, the participants studied 10 lists of 10 pairs of Swahili-English vocabulary pairs. After each study list, they took an initial test on all 10 items. In the unsuccessful-retrieval-with-feedback condition, a final cued-recall test occurred after every two lists (20 pairs). In the successful-retrieval condition, the final cued-recall test occurred after all 10 lists and covered all 100 pairs. (The reason for this difference in procedure is that pilot testing in the unsuccessful-retrieval condition showed that performance was at floor levels when participants studied all 10 lists before a final test; hence, we tested them after every two lists in this condition. This issue is described more fully below.) The picture manipulation in the initial test occurred immediately after either a successful retrieval or an unsuccessful retrieval with feedback. During the initial test, participants were not forced to supply an answer (i.e., they could leave the response field blank), and they had as much time as they wanted to enter their response.

In the unsuccessful-retrieval-with-feedback condition, all responses (both correct and incorrect) were immediately followed by a feedback presentation of the Swahili-English pair for $3 \mathrm{~s}$. A commission error was logged if the participant typed in an incorrect response, and an omission error was logged if the participant left the response field blank or typed in "don't know." After the feedback was shown, participants were presented with one of the three postfeedback picture manipulations. If the retrieval attempt had been unsuccessful (no response was given or the response was incorrect), the feedback was followed by a blank screen, a neutral picture, or a negatively valenced picture. If the response was correct, feedback was followed by either a blank screen or a neutral picture. In the unsuccessful condition, a negative picture never followed a correctly answered question. In the analysis of final test performance, we considered only items that were not successfully retrieved during this initial test.

In the successful-retrieval condition, feedback was not presented. As in the postretrieval conditions in Experiment 1 , a blank screen, a neutral picture, or a negative picture was presented immediately after a successful retrieval. Incorrect items were followed by either a blank screen or a neutral picture. In the analysis of final test performance, we considered only items that were answered correctly during this initial test. This procedure was identical to the negativepicture postretrieval condition in Experiment 1 and replicated that used in the Finn and Roediger (2011) experiments.

After the initial test phase, participants completed another minute of math before the next set of 10 pairs was presented for study. In the unsuccessful-retrieval-with-feedback condition, the final recall test occurred after every two lists, and thus on the previous 20 items. This was done to avoid floor effects. Though the entire pools of pairs studied and tested were identical for the unsuccessful and successful conditions, items in the unsuccessful-retrieval-with-feedback condition represented a more difficult set than those in the successful-retrieval condition, as they were, by definition, those items that could not be retrieved. Pilot work showed that subjects in the unsuccessful-retrieval-with-feedback condition were at floor when we presented 100 items before the final test. In the successful-retrieval condition, after all 10 lists had been studied and tested, all 100 items were randomized and presented for a final cued-recall test. (Ceiling effects would have occurred had we tested after every two lists, as in the unsuccessful-retrieval condition.) The lag to test between an item's presentation in the initial study-test phase and the final test phase was approximately $30 \mathrm{~min}$ in the successful-retrieval condition and approximately $10 \mathrm{~min}$ in the unsuccessful-retrieval-with-feedback condition. No distractor task intervened between the initial study-test phase and the final test phase.

\section{Results}

Initial test performance On average, participants in the successful-retrieval condition correctly recalled $.39(S E=.02)$ of the items in the list and those in the unsuccessful-retrievalwith-feedback condition recalled $.30(S E=.01)$ of the items in the list. This difference was significant, $t(205)=3.65, p<.001$. We have no interpretation of this difference, and it does not cloud interpretation of the results of primary interest, because 
those results are based on conditional recall of one item per list in which an item was retrieved (in the successful-retrieval condition) or not retrieved (in the unsuccessful retrieval condition).

Final test performance As in the postretrieval conditions in Experiment 1, final test performance for the successfulretrieval group was calculated using items that had been successfully retrieved on the initial test and were followed by one of the three within-participant picture manipulation conditions. Final test performance for the unsuccessfulretrieval-with-feedback group was analyzed using items that had been answered incorrectly on the initial test and were followed by one of the three within-participant picture manipulation conditions. A preliminary analysis found no interactions with normative item difficulty, and thus the following analyses were collapsed over this variable.

The final test performance data are depicted in Fig. 2, which shows that the negative pictures enhanced later recall (relative to the neutral-picture and blank conditions) after both successful retrieval attempts and unsuccessful retrieval attempts with feedback. A 2 (retrieval condition) $\times 3$ (picture event type: blank screen, neutral picture, or emotional picture) mixed-measures ANOVA revealed a main effect of event type, $F(2,404)=16.71, M S E=.02, p<.001, \eta_{\mathrm{p}}{ }^{2}=$ .08. The overall performance was better for items that had been followed by an emotional picture $(M=.48, S E=.01)$ than for items that had been followed by either a blank screen $(M=.42, S E=.01)$ or neutral picture $(M=.41$, $S E=.01)$. There was also a main effect of experimental

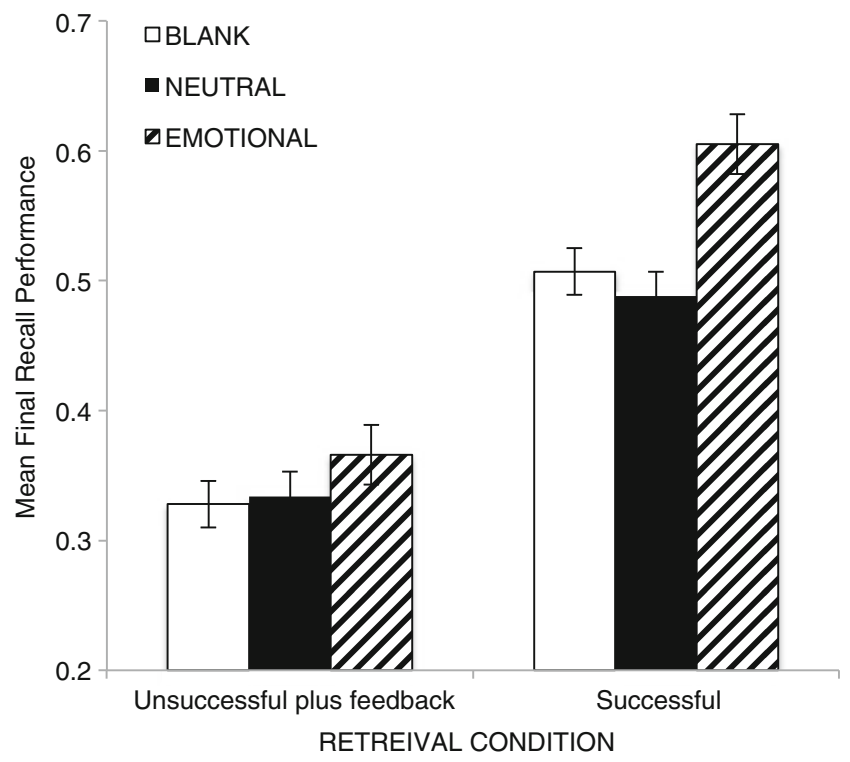

Fig. 2 Mean proportions correct on the final recall test for items answered incorrectly and followed by feedback (unsuccessful-retrieval-with-feedback condition) and for items answered correctly on the initial test (successful condition) in each experimental condition in Experiment 2. Error bars depict the standard errors of the means condition, $F(1,202)=66.25, M S E=.08, p<.001, \eta_{\mathrm{p}}{ }^{2}=.25$. Overall, final test performance in the successful condition $(M=.53, S E=.02)$ was better than performance in the unsuccessful-retrieval-with-feedback condition $(M=.34$, $S E=.02)$. This is not surprising, given that the items in the successful-retrieval condition had been recalled correctly during the previous test, while the items analyzed in the unsuccessful-retrieval-with-feedback condition were, by design, those items that had not been retrieved previously, and were therefore more difficult items. This outcome occurred despite the fact that the retention interval was longer for items that had been successfully retrieved (tested after all 10 lists) than for those with unsuccessful retrieval (tested after only two lists).

The magnitude of the effect (measured by comparing the average difference between the negative-picture and blank and the negative-picture and neutral-picture conditions) was numerically larger in the successful condition $(+.11$ vs. +.04 for the unsuccessful-with-feedback and successful groups, respectively). This difference in effect magnitudes was confirmed by a significant interaction between retrieval condition and picture manipulation, $F(2,404)=4.29, M S E=.02$, $p<.001, \eta_{\mathrm{p}}{ }^{2}=.02$. Pairwise comparisons between the picture conditions for each experimental group found that in both groups, participants' final test performance was significantly better for items followed by a negative picture than for items followed by either a neutral picture [unsuccessful with feedback, $t(102)=2.24, p<.05$, vs. successful, $t(100)=4.07, p<.001$ ] or a blank screen [unsuccessful with feedback, $t(102)=2.56, p<.05$, vs. successful, $t(100)=$ $3.70, p<.001]$. There was no significant difference in performance between the blank and neutral conditions in either the unsuccessful-retrieval-with-feedback $(t<1)$ or the successful-retrieval $[t(102)=1.11, p>.05]$ group.

Initial test performance in the unsuccessful-retrievalwith-feedback condition, conditionalized by error type (omission or commission) In the following analyses, we considered the errors in the unsuccessful-retrieval-withfeedback condition in more detail. If the cue was not recognized or if it brought nothing to mind during the initial test, the participant might not have attempted retrieval (in the former case) or might have truncated processing during retrieval (in the latter case), resulting in omission of a response. A commission error could result if the cue was recognized and retrieval was attempted, but the process ended with generation of an erroneous response. We compared final test performance in the unsuccessful-retrieval condition, conditionalized by whether the errors made during the initial test had been omission or commission errors. Despite the fact that all items were given feedback after an unsuccessful retrieval, final recall performance was better when a commission error had been generated $(M=.38, S E=$ 
$.02)$ than when an omission error had been made $(M=.26$, $S E=.02), t(102)=4.84, p<.001$.

An omission error might indicate that subjects did not engage in active retrieval to the same extent that they did with commission errors. If so, we would predict that the enhancing effect of negative pictures would be smaller following an omission error rather than a commission error. Across all participants, there was no interaction between error type (omission vs. commission) and picture event type, $F(1$, $112)=1.82, p>.05$. However, participants who did not show the enhancement would not be expected to show any differential effects in an analysis of the omission and commission errors; if they did not show the effect, item type could not have been a factor. Considering only the participants who did show the overall effect, we investigated further by examining differences in the enhancing effect following omission and commission errors. We selected only those subjects who showed a positive numerical difference between the negative-picture condition and both the blank and the neutral-picture conditions; this process reduced the number of subjects included in the analysis from 104 to 62 . As can be seen in Fig. 3, with this more restrictive analysis, we did find a stronger enhancing effect in the case of commission errors relative to omission errors. A 2 (error type: omission or commission) $\times 3$ (picture event type: blank screen, neutral picture, or negative picture) repeated measures ANOVA revealed that final test performance was better following a commission error $(M=.42$, $S E=.04)$ than following an omission error $(M=.33, S E=$ $.03), F(1,31)=8.68, M S E=.05, p<.01, \eta_{\mathrm{p}}{ }^{2}=.22$. Of course, performance was better for the items followed by negative pictures, $F(2,62)=15.13, M S E=.02, p<.001, \eta_{\mathrm{p}}{ }^{2}=.33$.

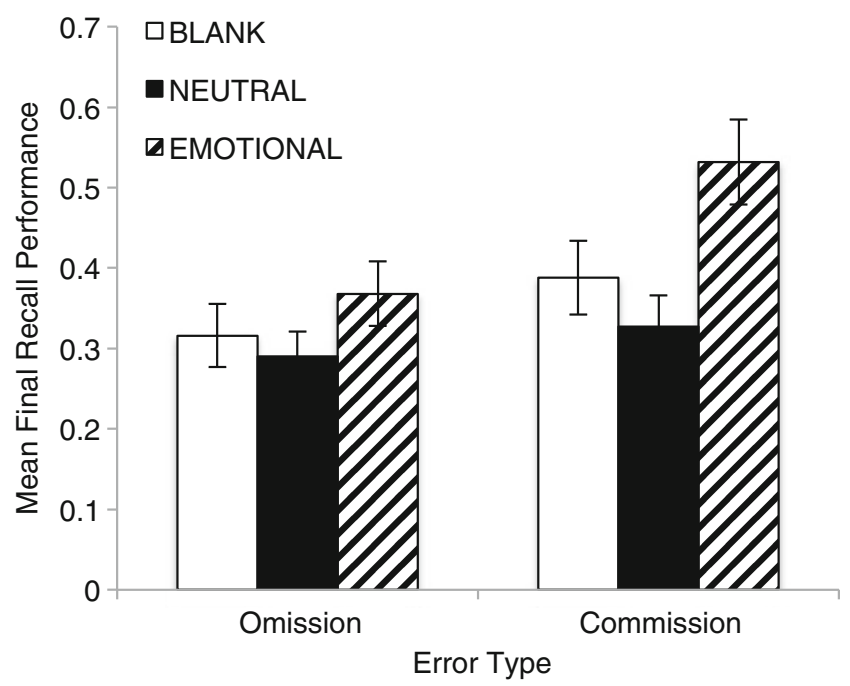

Fig. 3 Mean proportions correct on the final recall test for omission errors and commission errors for participants who showed an enhancement from negative pictures in the unsuccessful-retrieval-with-feedback condition in Experiment 2. Error bars depict the standard errors of the means
However, the enhancing effect of negative pictures was heightened for commission errors, as can be seen in Fig. 3, and as was indicated by a significant interaction between error type and picture manipulation, $F(2,62)=3.05, M S E=.02, p=$ $.05, \eta_{\mathrm{p}}{ }^{2}=.09$. To assess the locus of the interaction, post hoc comparisons of the picture manipulation conditions were computed separately for omission and commission errors. For commission errors, performance in the negative-picture condition was significantly better than performance in either the neutral-picture or the blank condition (smallest $t=2.98, p \mathrm{~s}<.01$ ), with no difference between the latter two conditions, $t(31)=1.81$, $p>.05$. With omission errors, however, only the difference between the negative and neutral pictures was significant, $t(31)=3.33, p<.01$, with the difference between the negative picture and a blank screen being not significant, $t(31)=1.34, p>.05$. There was no difference between the blank and neutral conditions $(t<1)$.

Semantic relationship between commission errors and target response Commission errors corrected by feedback may have been recalled better on a later test than were omission errors followed by feedback because of a more robust engagement of retrieval processing and activation of related semantic content, which in turn may have facilitated learning of the corrective feedback (see also Kornell et al., 2009). We assessed final test performance as a function of the semantic relationship between the original commission error and the target response, to test the hypothesis that making a semantically related commission error facilitated learning of feedback, as compared to when the commission error was not semantically related. Latent semantic analysis (LSA; Landauer \& Dumais, 1997, and see http://cwl-projects.cogsci.rpi.edu/ $\mathrm{msr} /$ ) was used to determine the association strength between the error that was generated and the target item (see also Metcalfe \& Finn, 2011). The LSA cosine value of every commission error and target pair was obtained, with higher values indicating a closer contextual relationship between the target and the commission error. (If an error was not a word, or could not be found in the database, it was eliminated from the analysis.) For each participant, the median split of LSA error to target values was calculated, with numbers above the median split classified as high-LSA items and numbers below the split classified as low-LSA items. The final test performance for items answered incorrectly on the initial test was significantly greater for high-LSA-value items, $M=.43$, $S E=.03$, than for low-LSA-value items, $M=.35, S E=.02$, $t(94)=3.02, p<.01$. This finding indicates that the strength of the semantic relationship between the commission error and the target influenced the probability of error correction following feedback. 


\section{Discussion}

Experiment 2 demonstrated that when negative emotional pictures followed successful retrieval, later recall was enhanced, thus replicating the findings of Finn and Roediger (2011) and of Experiment 1. More importantly, the results also showed that retention was enhanced when negative pictures followed unsuccessful retrieval with feedback, particularly when the participants made commission errors. The demonstration of a robust enhancing effect with commission errors suggests that the interval following a commission error with feedback may involve a period of reconsolidation that is similar to that following a successful retrieval.

\section{General discussion}

Two experiments showed that attempting retrieval and producing an initial answer is critical for the enhancing effect from negative pictures. We replicated the findings of Finn and Roediger (2011) in Experiment 1 and Experiment 2 by showing that when a negative emotional picture is presented immediately after a successful retrieval, later test performance is enhanced as compared to when a neutral picture or a blank screen is shown instead. We extended this finding by showing that successful retrieval is not necessary to show the enhancing effect of negative arousal from pictures on later recall, but that attempting retrieval does seem critical (because of the different effects on commission vs. omission errors, discussed below).

Experiment 1 provided converging evidence in support of the hypothesis that active retrieval is required to show the enhancing effect of negative pictures after retrieval. When the experimental manipulation was given just prior to successful retrieval - that is, before a retrieval attempt had been initiated - no positive effect from negative pictures occurred in later recall. This result also helps to rule out a distinctiveness account of our results. If the distinctiveness of the emotional pictures were driving the enhancing effect, the effect should also have emerged when the distinctive negative items were presented just prior to a successful retrieval. Similarly, the enhancing effect should have emerged in the positive postretrieval condition. Neither outcome occurred.

In Experiment 2, when an unsuccessful attempt was followed by corrective feedback (and a negative picture), enhancement on a later test was observed; however, the effect was much more pronounced after commission errors than after omission errors. Several notable differences were observed between omission and commission errors. First, overall recall was better on the final test when a commission error had been made on the initial test, rather than an omission error. Both groups had received corrective feedback during the intervening test, so that factor was nominally held constant. Apparently, when an active retrieval attempt was made that resulted in a commission error, the subsequent feedback was processed more effectively, resulting in superior performance on the final test (see also Kornell et al., 2009; Richland et al., 2009; see also McDermott \& Arnold, 2010). We propose that the better recall following commission errors than following omission errors indicates the engagement of more robust retrieval processing and the activation of related semantic content, which in turn facilitated learning of the corrective feedback. The finding of superior performance when commission errors were highly related to the target supports this claim. Of course, at the moment, this interpretation represents a hypothesis that awaits further testing, but it is consistent with our data.

The superior performance on the final test for items that resulted in commission errors rather than omission errors may also be related to the "hypercorrection effect," which refers to the finding that high-confidence errors are more easily corrected than low-confidence errors (see, e.g., Butterfield \& Metcalfe, 2001, 2006). High-confidence errors are generally made when the semantic or conceptual domain of the cue is familiar and/or the target is partially known (Butterfield \& Metcalfe, 2006; Metcalfe \& Finn, 2011). According to one explanation of the hypercorrection effect, less new learning is needed when the domain is familiar, which results in hypercorrection of high-confidence errors as compared to low-confidence errors (Metcalfe \& Finn, 2011). In Experiment 2, a participant's willingness to generate a commission error may have signaled familiarity with the cue or partial semantic access to the target, and potentially signaled a high-confidence error. Indeed, when a commission error was semantically related to the target, more errors were corrected on the final test. Finally, Butterfield and Metcalfe (2006) and Fazio and Marsh (2009) have suggested that highconfidence errors increase attention to feedback. Consistent with both the retrieval-processing account and the hypercorrection account, such familiarity and increased attention would promote favorable conditions for error correction when feedback was provided.

A second noteworthy finding was that the enhancing effect of presenting negative pictures was much diminished when an omission error had been made. Performance on the final test for omission errors did not reliably differ across all of the conditions. The fact that enhancement occurred chiefly after commission errors agrees well with the finding that reconsolidation processes are triggered when there is a mismatch between a predicted and an actual event (Lee, 2009; Nader \& Hardt, 2009; Pedreira, Perez-Cuesta, \& Maldonado, 2004). In the case of commission errors - which we, and others, have argued indicate familiarity with the cue - participants may be surprised to find that the feedback does not match the answer that they had provided (see also Rescorla \& Wagner, 1972). This mismatch between the commission error 
and the feedback is probably important for initiating reconsolidation of the correct cue-target association. In addition, the fact that the positive effect of negative pictures occurs after commission but not after omission errors provides additional support for our claim that an active retrieval attempt benefits long-term retention and that the testing effect can be increased by manipulations given after retrieval (or after a retrieval attempt involving a commission error and feedback). Thus, reconsolidation is probably involved in both these cases.

Why might memories become labile after reactivation and require a period of reconsolidation? Addressing this question seems critical if we are to understand the significance of postretrieval processing for learning (as well as for modification of traumatic and nonadaptive memories). Two prominent theories have been proposed about the functional role of reconsolidation (see Alberini, 2011, for a review). One hypothesis suggests that the function of reconsolidation is to strengthen and maintain memory traces (e.g., Sara, $2000 \mathrm{~b}$ ), to keep memories in an accessible state (as does repeated testing). The second idea proposes that reconsolidation allows for updating of an established trace with new information (e.g., Dudai, 2006; Forcato et al., 2007; Lee, 2009; Lewis, 1979; Nader et al., 2000), which can lead to negative effects when the new information undermines or conflicts with the original encoding (Hupbach et al., 2007; Loftus \& Palmer, 1974). These theories are not mutually exclusive, and decisive evidence showing whether one hypothesis is to be favored over the other is not yet at hand (Alberini, 2011). The function of retrieval-induced reconsolidation has yet to be determined.

Our results suggest roles for reconsolidation in both strengthening and updating episodic memory. Our finding showing enhancement from a negative picture given after effortful retrieval suggests that episodic retrieval and the attendant postretrieval processes are important for maintaining access to memories under the right conditions. According to Lee (2009), updating via reconsolidation maintains the "predictive and adaptive relevance" of memories (p. 413). An updating function may have particular relevance for the effective correction of errors. Our result showing enhancement from negative pictures when commission errors were followed by feedback suggests that reconsolidation may also have an updating function; the period of postretrieval processing fosters error correction via integration of the retrieved cue with the correct target information.

One potential limitation of the present set of experiments, as well as of those reported by Finn and Roediger (2011), is that the designs of the experiments all made use of the oddball technique, in which an emotional item is presented at most once per block of 10 neutral pictures or blanks. Although the original Finn and Roediger findings and those reported here have allowed us to argue strongly against a distinctiveness interpretation of the results, future studies should be directed at establishing whether the effect is dependent on the use of the oddball technique or can be obtained using a more balanced design. One approach might be to equate the picture event conditions in a block. It is possible, however, that the basic effect is dependent on using the oddball paradigm. The occurrence of an emotional oddball may signal relevance (e.g., Schupp et al., 2000), which may initiate adaptive modification of the retrieved information. These possibilities await empirical testing.

\section{Conclusion}

According to Tronson and Taylor (2007), "Demonstrating reconsolidation not only requires evidence of modification of a previously consolidated memory, but also evidence that, in the absence of retrieval the memory remains unmodified by the experimental manipulation" (p. 264). The results of the present experiments, as well as those of Finn and Roediger (2011), meet this criterion and demonstrate the behavioral dynamism and plasticity of retrieved memories. The findings presented here implicate the period immediately following retrieval as playing an important role in the later retention of that information. We propose that the benefits from retrieval practice can be characterized by the processes involved in attempting to retrieve (which probably always makes future retrieval more likely), but also by the processes occurring in the interval following reactivation of the memory. The latter processes involve reconsolidation and permit memories to be updated with new information.

Author note Support for this research was provided by a James S. McDonnell Foundation 21st Century Science Initiative Grant: Bridging Brain, Mind and Behavior/Collaborative Award. We thank Sam Barnes for help with the data collection.

\section{References}

Ackil, J. K., \& Zaragoza, M. S. (1998). Memorial consequences of forced confabulations: Age differences in susceptibility to false memories. Developmental Psychology, 34, 1358-1372.

Alberini, C. M. (2011). The role of reconsolidation in the dynamic process of long-term memory formation and storage. Frontiers in Behavioral Neuroscience, 5. Retrieved from www.frontiersin.org/ behavioral_neuroscience/10.3389/fnbeh.2011.00012/abstract

Baddeley, A. D., \& Wilson, B. A. (1994). When implicit learning fails: Amnesia and the problem of error elimination. Neuropsychologia, $32,53-68$.

Barnes, J. M., \& Underwood, B. J. (1959). "Fate" of first-list associations in transfer theory. Journal of Experimental Psychology, 58, 97-105. doi:10.1037/h0047507

Begg, I., \& Snider, A. (1987). The generation effect: Evidence for generalized inhibition. Journal of Experimental Psychology: 
Learning, Memory, and Cognition, 13, 553-563. doi:10.1037/ 0278-7393.13.4.553

Benjamin, A. S., Bjork, R. A., \& Schwartz, B. L. (1998). The mismeasure of memory: When retrieval fluency is misleading as a metamnemonic index. Journal of Experimental Psychology: General, 127, 55-68. doi:10.1037/0096-3445.127.1.55

Bjork, R. A. (1975). Retrieval as a memory modifier. In R. Solso (Ed.), Information processing and cognition: The Loyola Symposium (pp. 123-144). Hillsdale, NJ: Erlbaum.

Blaiss, C. A., \& Janak, P. H. (2006). Post-training and post-reactivation administration of amphetamine enhances morphine conditioned place preference. Behavioral Brain Research, 171, 329-337.

Bustos, S. G., Maldonado, H., \& Molina, V. A. (2009). Midazolam disrupts fear memory reconsolidation. Neuropsychopharmacology, 34, 446-57.

Butler, A. C., Karpicke, J. D., \& Roediger, H. L., III. (2007). The effect of type and timing of feedback on learning from multiple-choice tests. Journal of Experimental Psychology: Applied, 13, 273-281. doi:10.1037/1076-898X.13.4.273

Butler, A. C., \& Roediger, H. L., III. (2007). Testing improves longterm retention in a simulated classroom setting. European Journal of Cognitive Psychology, 19, 514-527.

Butterfield, B., \& Metcalfe, J. (2001). Errors committed with high confidence are hypercorrected. Journal of Experimental Psychology: Learning, Memory, and Cognition, 27, 1491-1494.

Butterfield, B., \& Metcalfe, J. (2006). The correction of errors committed with high confidence. Metacognition and Learning, 1, $1556-1623$.

Cacioppo, J. T., \& Gardner, W. L. (1999). Emotion. Annual Review of Psychology, 50, 191-214.

Cahill, L., \& McGaugh, J. L. (1998). Mechanisms of negative emotional arousal and lasting declarative memory. Trends in Neurosciences, 21, 294-299.

Carpenter, S. K., \& DeLosh, E. L. (2005). Application of the testing and spacing effects to name learning. Applied Cognitive Psychology, 19, 619-636.

Carrier, M., \& Pashler, H. (1992). The influence of retrieval on retention. Memory \& Cognition, 20, 633-642. doi:10.3758/ BF03202713

Carretié, L., Mercado, F., Tapia, M., \& Hinojosa, J. A. (2001). Emotion, attention, and the "negativity bias", studied through eventrelated potentials. International Journal of Psychophysiology, 41, $75-85$.

Clare, L., \& Jones, R. S. P. (2008). Errorless learning in the rehabilitation of memory impairment: A critical review. Neuropsychology Review, 18, 1-23.

Coccoz, V., Maldonado, H., \& Delorenzi, A. (2011). The enhancement of reconsolidation with a naturalistic mild stressor improves the expression of a declarative memory in humans. Neuroscience, $185,61-72$.

Craik, F. I. M., \& Tulving, E. (1975). Depth of processing and the retention of words in episodic memory. Journal of Experimental Psychology: General, 104, 268-294. doi:10.1037/00963445.104.3.268

Cunningham, D., \& Anderson, R. C. (1968). Effects of practice time within prompting and confirmation presentation procedures on paired associate learning. Journal of Verbal Learning and Verbal Behavior, 7, 613-616.

Delplanque, S., Lavoie, M., Hot, P., Silvert, L., \& Sequeira, H. (2004). Modulation of cognitive processing by emotional valence studied through event-related potentials in humans. Neuroscience Letters, 356,1 .

DeVietti, T. L., Conger, G. L., \& Kirkpatrick, B. R. (1977). Comparison of the enhancement gradients of retention obtained with stimulation of the mesencephalic reticular formation after training or memory reactivation. Physiology and Behavior, 19, 549-554.
Dudai, Y. (2004). The neurobiology of consolidations, or, how stable is the engram? Annual Review of Psychology, 55, 51-86. doi:10.1146/annurev.psych.55.090902.142050

Dudai, Y. (2006). Reconsolidation: The advantage of being refocused. Current Opinion in Neurobiology, 16, 174-178.

Fazio, L. K., \& Marsh, E. J. (2009). Surprising feedback improves later memory. Psychonomic Bulletin \& Review, 16, 88-92.

Finn, B., \& Metcalfe, J. (2010). Scaffolding feedback to maximize long-term error correction. Memory \& Cognition, 38, 951-961.

Finn, B., \& Roediger, H. L., III. (2011). Enhancing retention through reconsolidation: Negative emotional arousal following retrieval enhances later recall. Psychological Science, 22, 781-786. doi:10.1177/0956797611407932

Finn, B., Rosenzweig, E. Q., \& Roediger, H. L., III. (2012). Which types of arousing stimuli produce enhancement following retrieval? Manuscript in preparation

Forcato, C., Burgos, V. L., Argibay, P. F., Molina, V. A., Pedreira, M. E., \& Maldonado, H. (2007). Reconsolidation of declarative memory in humans. Learning and Memory, 14, 295-303.

Frenkel, L., Maldonado, H., \& Delorenz, A. (2005). Memory strengthening by a real-life episode during reconsolidation: An outcome of water deprivation via brain angiotensin II. European Journal of Neuroscience, 22, 1757-1766.

Frenkel, L., Suarez, L. D., Maldonado, H., \& Delorenzi, A. (2010). Angiotensin modulates long-term memory expression but not long-term memory storage in the crab Chasmagnathus. Neurobiology of Learning and Memory, 94, 509-520.

Gardiner, J. M., Craik, F. I. M., \& Bleasdale, F. A. (1973). Retrieval difficulty and subsequent recall. Memory \& Cognition, 1, 213216.

Glanzer, M., \& Cunitz, A. R. (1966). Two storage mechanisms in free recall. Journal of Verbal Learning and Verbal Behavior, 5, 351360. doi:10.1016/S0022-5371(66)80044-0

Glisky, E. L., Schacter, D. L., \& Tulving, E. (1986). Learning and retention of computer related vocabulary in memory impaired patients: Method of vanishing cues. Journal of Clinical and Experimental Neuropsychology, 8, 292-312.

Glover, J. A. (1989). The "testing" phenomenon: Not gone but nearly forgotten. Journal of Educational Psychology, 81, 392-399.

Gordon, W. C., \& Spear, N. E. (1973). The effect of reactivation of a previously acquired memory on the interaction between memories in the rat. Journal of Experimental Psychology, 99, 349-355.

Grimaldi, P. J., \& Karpicke, J. D. (2010, November). When and why does pretesting enhance subsequent encoding? Poster presented at the 51st Annual Meeting of the Psychonomic Society. St. Louis, MO.

Hastie, R., Landsman, R., \& Loftus, E. F. (1978). Eyewitness testimony: The dangers of guessing. Jurimetrics Journal, 19, 1-8.

Heusler, B. J., \& Metcalfe, J. (2010, November). When does initial retrieval failure lead to later success? Poster presented at the 51st Annual Meeting of the Psychonomic Society, St. Louis, MO

Hunt, R. R. (2006). The concept of distinctiveness in memory research. In R. R. Hunt \& J. B. Worthen (Eds.), Distinctiveness and memory (pp. 3-25). New York, NY: Oxford University Press.

Hupbach, A., Gomez, L., Hardt, O., \& Nadel, R. (2007). Reconsolidation of episodic memories: A subtle reminder triggers integration of new information. Learning and Memory, 14, 47-53.

Inda, M. C., Muravieva, E., \& Alberini, C. M. (2011). Memory retrieval and the passage of time: From reconsolidation and strengthening to extinction. Journal of Neuroscience, 31, 1635-1643.

Ito, T. A., Larsen, J. T., Smith, N. K., \& Cacioppo, J. T. (1998). Negative information weighs more heavily on the brain: The negativity bias in evaluative categorizations. Journal of Personality and Social Psychology, 75, 887-900.

Izawa, C. (1971). The test trial potentiating model. Journal of Mathematical Psychology, 8, 200-224. doi:10.1016/0022-2496(71) 90012-5 
Jacoby, L. L., Shimizu, Y., Daniels, K. A., \& Rhodes, M. G. (2005). Modes of cognitive control in recognition and source memory: Depth of retrieval. Psychonomic Bulletin \& Review, 12, 852-857.

Kane, J. H., \& Anderson, R. C. (1978). Depth of processing and interference effects in the learning and remembering of sentences. Journal of Educational Psychology, 70, 626-635.

Kensinger, E. A. (2007). Negative emotion enhances memory accuracy: Behavioral and neuroimaging evidence. Current Directions in Psychological Science, 16, 213-218.

Kensinger, E. A. (2009). Emotional memory across the adult lifespan. New York, NY: Psychology Press.

Kessels, R. P. C., \& de Haan, E. H. F. (2003). Implicit learning in memory rehabilitation: A meta-analysis on errorless learning and vanishing cues methods. Journal of Clinical \& Experimental Neuropsychology, 25, 805-814.

Kornell, N., Hays, M. J., \& Bjork, R. A. (2009). Unsuccessful retrieval attempts enhance subsequent learning. Journal of Experimental Psychology: Learning, Memory, and Cognition, 35, 989-998. doi:10.1037/a0015729

Landauer, T. K., \& Dumais, S. T. (1997). A solution to Plato's problem: The latent semantic analysis theory of acquisition, induction, and representation of knowledge. Psychological Review, 104, 211-240. doi:10.1037/0033-295X.104.2.211

Lang, P. J., Bradley, M. M., \& Cuthbert, B. N. (2008). International affective picture system (IAPS): Affective ratings of pictures and instruction manual (Technical Report No. A8). Gainesville, FL: University of Florida, Center for Research in Psychophysiology.

Lee, J. L. C. (2009). Reconsolidation: Maintaining memory relevance. Trends in Neurosciences, 32, 413-420.

Lee, J. L. C., Milton, A. L., \& Everitt, B. J. (2006). Reconsolidation and extinction of conditioned fear: Inhibition and potentiation. Journal of Neuroscience, 26, 10051-10056.

Lewis, D. J. (1979). Psychobiology of active and inactive memory. Psychological Bulletin, 86, 1054-1083.

Loftus, E. F. (1996). Eyewitness testimony. Cambridge, MA: Harvard University Press.

Loftus, E. F., \& Palmer, J. C. (1974). Reconstruction of automobile destruction: An example of the inter-action between language and memory. Journal of Verbal Learning and Verbal Behavior, 13, $585-589$.

Marsh, E. J., Roediger, H. L., III, Bjork, R. A., \& Bjork, E. L. (2007). The memorial consequences of multiple-choice testing. Psychonomic Bulletin \& Review, 14, 194-199.

McDaniel, M. A., \& Masson, M. E. J. (1985). Altering memory representations through retrieval. Journal of Experimental Psychology: Learning, Memory, and Cognition, 11, 371-385.

McDaniel, M. A., Roediger, H. L., III, \& McDermott, K. B. (2007). Generalizing test-enhanced learning from the laboratory to the classroom. Psychonomic Bulletin \& Review, 14, 200-206.

McGaugh, J. L. (2000). Memory-A century of consolidation. Science, 287, 248-251. doi:10.1126/science.287.5451.248

McGaugh, J. L., \& Roozendaal, B. (2002). Role of adrenal stress hormones in forming lasting memories in the brain. Current Opinion in Neurobiology, 12, 205-210.

Metcalfe, J., \& Finn, B. (2011). People's correction of high confidence errors: Did they know it all along? Journal of Experimental Psychology: Learning, Memory, and Cognition, 37, 437-448.

Nader, K. (2003). Memory traces unbound. Trends in Neuroscience, $26,65-72$.

Nader, K., \& Hardt, O. (2009). A single standard for memory: The case for reconsolidation. Nature Reviews Neuroscience, 10, 224-234.

Nader, K., Schafe, G. E., \& LeDoux, J. E. (2000). Fear memories require protein synthesis in the amygdala for reconsolidation after retrieval. Nature, 406, 722-726.
Nelson, T. O., \& Dunlosky, J. (1994). Norms of paired-associate recall during multitrial learning of Swahili-English translation equivalents. Memory, 2, 325-335.

Pedreira, M. E., Perez-Cuesta, L. M., \& Maldonado, H. (2004). Mismatch between what is expected and what actually occurs triggers memory reconsolidation or extinction. Learning and Memory, 11, 579-585.

Przybyslawski, J., \& Sara, S. J. (1997). Reconsolidation of memory after its reactivation. Behavioural Brain Research, 84, 241-246.

Rescorla, R. A., \& Wagner, A. R. (1972). A theory of Pavlovian conditioning: Variations in the effectiveness of reinforcement and nonreinforcement. In A. H. Black \& W. F. Prokasy (Eds.), Classical conditioning II: Current research and theory (pp. 64 99). New York, NY: Appleton-Century-Crofts.

Richland, L. E., Kornell, N., \& Kao, L. S. (2009). The pretesting effect: Do unsuccessful retrieval attempts enhance learning? Journal of Experimental Psychology: Applied, 15, 243-257.

Rodriguez, W. A., Horne, C. A., \& Padilla, C. L. (1999). Effects of glucose and fructose on recently reactivated and recently acquired memories. Progress in Neuro-Psychopharmacolgy \& Biological Psychiatry, 23, 1285-1317.

Roediger, H. L., III, \& Butler, A. C. (2011). The critical role of retrieval practice in long-term retention. Trends in Cognitive Sciences, 15, 20-27. doi:10.1016/j.tics.2010.09.003

Roediger, H. L., III, \& Karpicke, J. D. (2006). Test-enhanced learning: Taking memory tests improves long-term retention. Psychological Science, 17, 249-255. doi:10.1111/j.1467-9280.2006.01693.x

Roediger, H. L., III, \& Marsh, E. J. (2005). The positive and negative consequences of multiple-choice testing. Journal of Experimental Psychology: Learning, Memory and Cognition, $31,1155-1159$.

Roediger, H. L., III, Wheeler, M. A., \& Rajaram, S. (1993). Remembering, knowing and reconstructing the past. In D. L. Medin (Ed.), The psychology of learning and motivation: Advances in research and theory (Vol. 30, pp. 97-134). New York, NY: Academic Press.

Rohrbaugh, M., \& Riccio, D. C. (1970). Paradoxical enhancement of learned fear. Journal of Abnormal Psychology, 75, 210 216.

Roozendaal, B., Barsegyan, A., \& Lee, S. (2008). Adrenal stress hormones, amygdala activation, and memory for emotionally arousing experiences. Progress in Brain Research, 167, 7997.

Sara, S. J. (2000a). Retrieval and reconsolidation: Toward a neurobiology of remembering. Learning and Memory, 7, 73-84.

Sara, S. J. (2000b). Strengthening the shaky trace through retrieval. Nature Reviews Neuroscience, 1, 212-213.

Schiller, D., Monfils, M. H., Raio, C. M., Johnson, D. C., LeDoux, J. E., \& Phelps, E. A. (2010). Preventing the return of fear in humans using reconsolidation update mechanisms. Nature, 463, 49-53.

Schmidt, S. R. (1991). Can we have a distinctive theory of memory? Memory and Cognition, 19, 523-542.

Schmidt, S. R. (2002). Outstanding memories: The positive and negative effects of nudes on memory. Journal of Experimental Psychology: Learning, Memory, and Cognition, 28, 353361.

Schupp, H. T., Cuthbert, B. N., Bradley, M. M., Cacioppo, J. T., Ito, T., \& Lang, P. J. (2000). Affective picture processing: The late positive potential is modulated by motivational relevance. Psychophysiology, 37, 257-261.

Shema, R., Haramati, S., Ron, S., Hazvi, S., Chen, A., Sackton, T. C., \& Dudai, Y. (2011). Enhancement of consolidated long-term memory by overexperession of protein kinase Mzeta in the neocortex. Science, 331, 1207-1210. 
Skinner, B. F. (1961). Teaching machines. Scientific American, 205, 90-102.

Slamecka, N. J., \& Fevreiski, J. (1983). The generation effect when generation fails. Journal of Verbal Learning and Verbal Behavior, 22, 153-163. doi:10.1016/S0022-5371(83)90112-3

Strange, B. A., Hurlemann, R., \& Dolan, R. J. (2003). An emotioninduced retrograde amnesia in humans is amygdala- and betaadrenergic-dependent. Proceedings of the National Academy of Sciences, 100, 13626-13631.
Suzuki, A., Josselyn, S. A., Frankland, P. W., Masushige, S., Silva, A. J., \& Kida, S. (2004). Memory reconsolidation and extinction have distinct temporal and biochemical signatures. Journal of Neuroscience, 24, 4787-4795. doi:10.1523/JNEUROSCI.5491-03.2004

Tronson, N. C., \& Taylor, J. R. (2007). Molecular mechanisms of memory reconsolidation. Nature Reviews Neuroscience, 8, 262275. doi:10.1038/nrn2090

Tulving, E. (1969). Retrograde amnesia in free recall. Science, 164, 88-90. doi:10.1126/science.164.3875.88 